\title{
Correction: Maternal age-specific risk for trisomy 21 based on the clinical performance of NIPT and empirically derived NIPT age- specific positive and negative predictive values in Japan
}

\author{
Takahiro Yamada $\mathbb{1}^{1,2,13} \cdot$ Akihiko Sekizawa ${ }^{1,3}$ - Yosuke Fujii $\cdot$ Tatsuko Hirose ${ }^{1,3} \cdot$ Osamu Samura ${ }^{1,5}$. \\ Nobuhiro Suzumori ${ }^{1,6} \cdot{\text { Kiyonori Miura } \mathbb{D}^{1,7} \cdot \text { Hideaki Sawai }^{1,8} \cdot \text { Fumiki Hirahara }^{1,9} \cdot \text { Jun Murotsuki }}^{1,10}$. \\ Yoshimasa Kamei ${ }^{1,11} \cdot$ Haruhiko Sago ${ }^{1,12} \cdot$ Japan NIPT consortium ${ }^{* 1}$
}

Published online: 1 August 2018

(c) The Author(s) under exclusive licence to The Japan Society of Human Genetics 2018

Correction to: Journal of Human Genetics advance online publication 30 May 2018: https://www.nature.com/articles/ s10038-018-0453-8

Since the advance online publication of this article, the authors of the above paper have noticed errors in the list of authors and affiliations. The article with correct author information now appears in this issue.

The online html and pdf versions have also been rectified.

The authors would like to apologize for the error.

The correct authors and affiliations are listed as follows:

Takahiro Yamada ${ }^{1,2,13}$, Akihiko Sekizawa ${ }^{1,3}$, Yosuke Fujii $^{4}$, Tatsuko Hirose ${ }^{1,3}$, Osamu Samura ${ }^{1,5}$, Nobuhiro Suzumori $^{1,6}$, Kiyonori Miura ${ }^{1,7}$, Hideaki Sawai ${ }^{1,8}$, Fumiki Hirahara $^{1,9}$, Jun Murotsuki ${ }^{1,10}$, Yoshimasa Kamei ${ }^{1,11}$, Haruhiko Sago ${ }^{1,12}$, and Japan NIPT Consortium*1

1) Japan NIPT Consortium, Tokyo, Japan

2) Department of Obstetrics, Hokkaido University Graduate School of Medicine, Sapporo, Japan

3) Department of Obstetrics and Gynecology, The Showa University School of Medicine, Tokyo, Japan

4) Kyoto University Graduate School of Medicine, Kyoto, Japan

The original article can be found online at https://doi.org/10.1038/ s10038-018-0453-8.

*Study Collaborators of Japan NIPT Consortium are described separately

Takahiro Yamada

taka0197@kuhp.kyoto-u.ac.jp

Extended author information available on the last page of the article.
5) Department of Obstetrics and Gynecology, The Jikei University School of Medicine, Tokyo, Japan

6) Department of Obstetrics and Gynecology, Nagoya City University Graduate School of Medical Sciences, Nagoya, Japan

7) Department of Obstetrics and Gynecology, Nagasaki University Graduate School of Biomedical Sciences, Nagasaki, Japan

8) Department of Obstetrics and Gynecology, Hyogo College of Medicine, Nishinomiya, Japan

9) Department of Obstetrics and Gynecology, Yokohama City University Graduate School of Medicine, Yokohama, Japan

10) Department of Maternal and Fetal Medicine, Tohoku University Graduate School of Medicine, Miyagi-Children's Hospital, Sendai, Japan

11) Department of Obstetrics and Gynecology, Saitama Medical University, Saitama, Japan

12) Center of Maternal-Fetal, Neonatal and Reproductive Medicine, National Center for Child Health and Development, Tokyo, Japan

13) Present address: Clinical Genetics Unit, Kyoto University Hospital, 54 Shogoin-Kawaharacho, Sakyo-ku, Kyoto 606-8507, Japan

\section{Study collaborators of Japan NIPT Consortium:}

Takashi Kaji (The University of Tokushima Faculty of Medicine), Masanobu Ogawa (National Hospital Organization Kyushu Medical Center), Keiichi Matsubara (Ehime University School of Medicine), Haruka Hamanoue (Yokohama City University Graduate School of Medicine), Akimune Fukushima (Iwate Medical University School of Medicine), Masayuki Endo (Osaka University), Kazufumi Haino (Niigata University Medical and Dental Hospital), Hideaki Masuzaki (Nagasaki University Graduate School of 
Biomedical Sciences), Masaki Ogawa (Tokyo Women's Medical University Hospital), Shinya Tairaku (Kobe University Graduate School of Medicine), Masato Mizuuchi (Sapporo Medical University School of Medicine), Yoko Okamoto (Osaka Medical Center and Research Institute for Maternal and Child Health), Yukie Kawano (Oita University), Hisashi Masuyama (Okayama University Graduate School of Medicine), Hisao Osada (Chiba University Graduate School of Medicine), Taihei Tsunemi (Nara Medical University), Kazuhisa Maeda (Shikoku Medical Center for Children and Adults), Yasuyo Kasai (Japanese Red Cross Medical Center), Reiko Neki (National
Cerebral and Cardiovascular Center), Yukiko Katagiri (Toho University Omori Medical Center), Shunichiro Izumi (Tokai University School of Medicine), Setsuko Nakayama (Aiiku Hospital), Yuko Yokohama (Asahikawa Medical University), Masaya Hirose (Hyogo Prefectural Amagasaki General Medical Center), Kousuke Kawakami (National Hospital Organization Kokura Medical Center), Kiyotake Ichizuka (Showa University Northern Yokohama Hospital), Masakatsu Sase (Yamaguchi Grand Medical Center), Satoru Sakatsume (Dokkyo Medical University Koshigaya Hospital), Tomohiko Tsuruta (Kansai Rosai Hospital)

\section{Affiliations}

Takahiro Yamada $\circledast^{1,2,13}$ - Akihiko Sekizawa ${ }^{1,3}$. Yosuke Fujii ${ }^{4}$ Tatsuko Hirose ${ }^{1,3} \cdot$ Osamu Samura ${ }^{1,5}$. Nobuhiro Suzumori ${ }^{1,6} \cdot$ Kiyonori Miura $\unrhd^{1,7} \cdot$ Hideaki Sawai ${ }^{1,8}$ - Fumiki Hirahara ${ }^{1,9}$ - Jun Murotsuki ${ }^{1,10}$. Yoshimasa Kamei ${ }^{1,11} \cdot$ Haruhiko Sago ${ }^{1,12}$ Japan NIPT consortium ${ }^{* 1}$

1 Japan NIPT Consortium, Tokyo, Japan

2 Department of Obstetrics, Hokkaido University Graduate School of Medicine, Sapporo, Japan

3 Department of Obstetrics and Gynecology, The Showa University School of Medicine, Tokyo, Japan

4 Kyoto University Graduate School of Medicine, Kyoto, Japan

5 Department of Obstetrics and Gynecology, The Jikei University School of Medicine, Tokyo, Japan

6 Department of Obstetrics and Gynecology, Nagoya City University Graduate School of Medical Sciences, Nagoya, Japan

7 Department of Obstetrics and Gynecology, Nagasaki University Graduate School of Biomedical Sciences, Nagasaki, Japan

8 Department of Obstetrics and Gynecology, Hyogo College of
Medicine, Nishinomiya, Japan

9 Department of Obstetrics and Gynecology, Yokohama City University Graduate School of Medicine, Yokohama, Japan

10 Department of Maternal and Fetal Medicine, Tohoku University Graduate School of Medicine, Miyagi-Children's Hospital, Sendai, Japan

11 Department of Obstetrics and Gynecology, Saitama Medical University, Saitama, Japan

12 Center of Maternal-Fetal, Neonatal and Reproductive Medicine, National Center for Child Health and Development, Tokyo, Japan

13 Present address: Clinical Genetics Unit, Kyoto University Hospital, 54 Shogoin-Kawaharacho, Sakyo-ku, Kyoto 606-8507, Japan 